\title{
CONSEQUENCES OF PROPAGATING TORSION IN CONNECTION-DYNAMIC THEORIES OF GRAVITY*
}

\author{
Sean M. Carroll ${ }^{(1)}$ and George B. Field ${ }^{(2)}$ \\ ${ }^{(1)}$ Center for Theoretical Physics, Laboratory for Nuclear Science \\ and Department of Physics \\ Massachusetts Institute of Technology \\ Cambridge, Massachusetts 02139 \\ email: carroll@marie.mit.edu \\ ${ }^{(2)}$ Harvard-Smithsonian Center for Astrophysics \\ Cambridge, Massachusetts 02138 \\ email: field@cfa.harvard.edu
}

\begin{abstract}
We discuss the possibility of constraining theories of gravity in which the connection is a fundamental variable by searching for observational consequences of the torsion degrees of freedom. In a wide class of models, the only modes of the torsion tensor which interact with matter are either a massive scalar or a massive spin-1 boson. Focusing on the scalar version, we study constraints on the two-dimensional parameter space characterizing the theory. For reasonable choices of these parameters the torsion decays quickly into matter fields, and no long-range fields are generated which could be discovered by ground-based or astrophysical experiments.
\end{abstract}

CTP \# 2291

March 1994

gr-qc/9403058

* This work was supported in part by NASA under Grants no. NAGW-931 and NGT50850, by the National Science Foundation under grant PHY/92-06867, and by the U.S. Department of Energy (D.O.E.) under contract no. DE-FC02-94ER40818. 


\section{Introduction}

General relativity as formulated by Einstein describes the dynamics of a metric tensor field $g_{\mu \nu}$ and the response of matter to this metric. Covariant derivatives are taken with respect to the Christoffel connection on the tangent bundle. This is the unique metriccompatible and torsion-free connection, and is treated as a quantity derived from the metric. Despite the success of Einstein's theory in passing observational tests, there are strong indications that general relativity is incomplete - the prediction of singularities, and especially the difficulty of formulating a quantum theory. It is therefore natural to explore modifications of general relativity in which these problems may be overcome. One popular modification is to make the connection itself a fundamental variable in its own right, rather than a convenient expression for a certain function of the metric.

Such "connection-dynamic" theories can take different forms. A simple approach is the first-order or Palatini formulation of conventional gravity [1]. The action of this theory is that of general relativity, with the connection varied independently rather than given as a function of the metric. The resulting equations of motion lead to the usual expression for the connection in terms of the metric, with extra terms depending algebraically on the matter fields. Extensions of this procedure have been adopted in attempts to construct quantum versions of general relativity, including work in $(3+1)$ dimensions [2] and $(2+1)$ dimensions [3]. (For reviews and other approaches see [4].) The additional terms contributing to the connection in these theories are characterized by the torsion tensor; since no derivatives of the torsion appear in the Ricci scalar, the Palatini formulation leads to non-propagating torsion [5].

Once torsion has been introduced by taking the connection to be an independent variable, the restriction to non-propagating torsion arises as much from historical accident as from first principles. Just as the Einstein-Hilbert action provides dynamics for the metric degrees of freedom, it is straightforward to consider the addition of extra terms to the action which would provide dynamics for the torsion degrees of freedom.

A comparison with conventional gauge theories serves to illustrate why we believe that a dynamical torsion tensor is a natural expectation when the connection is treated as a variable independent from the metric. In gauge theories of an internal symmetry, the connection is specified by a non-gauge-invariant vector potential with an associated gauge-covariant tensor, the curvature or field strength. In contrast, the connection $\nabla$ on spacetime is associated with two tensors, the curvature and the torsion. ${ }^{1}$ We may specify the curvature and torsion of $\nabla$ in terms of their action on vector fields $X, Y$, and $Z$. (For

1 In general, the connection $\nabla$ on the tangent bundle is a $\operatorname{GL}(4, \mathbf{R})$ connection. When we restrict our attention to metric-compatible connections the group is reduced to $\mathrm{SO}(3,1)$. 
additional formulae see $[5,6]$.) The curvature, a $(1,3)$ tensor, is

$$
R(X, Y) Z \equiv \nabla_{X} \nabla_{Y} Z-\nabla_{Y} \nabla_{X} Z-\nabla_{[X, Y]} Z
$$

while the torsion, a $(1,2)$ tensor, is

$$
T(X, Y) \equiv \nabla_{X} Y-\nabla_{Y} X-[X, Y]
$$

In these expressions we use $\nabla_{X}$ to denote a covariant derivative in the direction along $X$, and $[X, Y]$ for the Lie bracket. $^{2}$ In a basis adapted to a set of coordinates $x^{\mu}$, we can decompose these tensors in terms of their components; thus the covariant derivative is

$$
\begin{aligned}
\left(\nabla_{X} Y\right)^{\lambda} & =X^{\mu} \nabla_{\mu} Y^{\lambda} \\
& =X^{\mu}\left(\partial_{\mu} Y^{\lambda}+\Gamma_{\mu \nu}^{\lambda} Y^{\nu}\right)
\end{aligned}
$$

while the Lie bracket is

$$
[X, Y]^{\mu} \equiv X^{\nu} \partial_{\nu} Y^{\mu}-Y^{\nu} \partial_{\nu} X^{\mu}
$$

The curvature tensor is then

$$
R_{\beta \mu \nu}^{\alpha}=\partial_{\mu} \Gamma_{\nu \beta}^{\alpha}-\partial_{\nu} \Gamma_{\mu \beta}^{\alpha}+\Gamma_{\mu \lambda}^{\alpha} \Gamma_{\nu \beta}^{\lambda}-\Gamma_{\nu \lambda}^{\alpha} \Gamma_{\mu \beta}^{\lambda},
$$

and the torsion tensor is

$$
\begin{aligned}
T_{\rho \sigma}{ }^{\mu} & =\Gamma_{\rho \sigma}^{\mu}-\Gamma_{\sigma \rho}^{\mu} \\
& =2 \Gamma_{[\rho \sigma]}^{\mu} .
\end{aligned}
$$

Thus, the curvature and torsion have a similar status as tensors which characterize a specified connection. Special relativity posits a spacetime connection for which both tensors vanish; the transition from special to general relativity may be thought of as allowing for the dynamics of a nonzero curvature, while constraining the torsion to vanish. From a point of view which takes the connection as an independent variable, this restriction seems somewhat arbitrary (although it is nevertheless possible, by judicious choice of Lagrangian, to make the torsion nonpropagating or even vanishing). We are therefore led to consider theories in which both the curvature and torsion are determined dynamically by the response of the metric and connection to matter fields.

2 As can be seen from (1.2), in the definition of torsion (unlike that of the curvature) a single vector field such as $X$ or $Y$ serves both as a direction in spacetime along which a covariant derivative is taken and as the object being differentiated. This is clearly only possible when the vector field is a section of the tangent bundle rather than an "internal" vector bundle; thus, the existence of torsion distinguishes the connection on the tangent bundle from the connections familiar from conventional gauge theories. 
The introduction of additional propagating degrees of freedom opens the possibility that such a theory could lead to observable deviations from general relativity. Experiments in the solar system and in binary pulsar 1913+16 offer strong evidence that the metric must not deviate too far from the form specified by Einstein's equations [7]. The situation with respect to torsion is less clear, as the literature contains various different proposals for what the dynamics of torsion could be.

Our goal in this paper is to determine whether there are any observational consequences of propagating torsion which are relatively independent of any specific gravitational model. To that end, we discuss possible actions for torsion and its interaction with matter fields such as those in the standard model of particle physics. In these theories we construct a free Lagrangian from powers and derivatives of the torsion, and couple "minimally" to matter through the covariant derivative. We find that there is only a small range of models possible without placing arbitrary restrictions on the dynamics. In these models only a single mode interacts with matter, either a massive scalar or a massive spin-1 field, and each model is parameterized by two constants with the dimensions of mass. In this paper we concentrate on the scalar theory, which is related to several different proposals found in the literature. We discuss what regions of parameter space are excluded by laboratory and astrophysical data. A reasonable expectation, however, would be for each of the two mass parameters to be of order the Planck scale; such a choice is a safe distance away from the regions excluded by experiment. We conclude that, while there are reasons to expect that the torsion degrees of freedom exist as propagating fields, there is no reason to expect any observable signature from torsion.

\section{Lagrangians}

Since our goal is to search for potentially observable consequences of a theory of gravity with propagating torsion, and not to construct the full theory itself, we shall limit our attention to the dynamics of torsion in a background spacetime with the Minkowski metric, $g_{\mu \nu}=\eta_{\mu \nu}$. (Actions for gravity with propagating torsion, but without couplings to matter, have been studied in $[8,9]$.) Furthermore, we shall not worry about the renormalizability of the torsion sector, since quantization of the full gravity theory is beyond our reach anyway. Nevertheless, we shall keep in mind known quantum effects (such as anomalies) in the matter sector. Thus, we are interested in theories defined by Lagrangians of the form

$$
\mathcal{L}=\mathcal{L}_{T}+\mathcal{L}_{I}+\mathcal{L}_{M}
$$

where $\mathcal{L}_{T}$ is the part of the Lagrangian containing only torsion fields, $\mathcal{L}_{M}$ is the matter Lagrangian, and $\mathcal{L}_{I}$ defines interactions between torsion and matter.

We turn first to the construction of $\mathcal{L}_{I}$, which involves an unavoidable ambiguity. In a 
spacetime with a metric, we may always decompose a metric-compatible connection (which is all we shall consider) into a torsion-free Christoffel piece plus a torsion-dependent piece. In terms of components we write

$$
\Gamma_{\mu \nu}^{\alpha}=\left\{\begin{array}{c}
\alpha \\
\mu \nu
\end{array}\right\}+\frac{1}{2}\left(T_{\mu \nu}{ }^{\alpha}-T_{\nu}{ }^{\alpha}{ }_{\mu}+T_{\mu \nu}^{\alpha}\right)
$$

where the Christoffel symbols $\left\{\begin{array}{c}\alpha \\ \mu \nu\end{array}\right\}$ are given by the familiar formula

$$
\left\{\begin{array}{c}
\alpha \\
\mu \nu
\end{array}\right\} \equiv \frac{1}{2} g^{\alpha \beta}\left(\partial_{\mu} g_{\nu \beta}+\partial_{\nu} g_{\beta \mu}-\partial_{\beta} g_{\mu \nu}\right) \text {. }
$$

The transformation properties of (2.3) are those of a connection, just as in conventional general relativity. Therefore the Christoffel covariant derivative

$$
\widetilde{\nabla}_{\mu} X^{\alpha} \equiv \partial_{\mu} X^{\alpha}+\left\{\begin{array}{c}
\alpha \\
\mu \lambda
\end{array}\right\} X^{\lambda}
$$

is a well-defined tensor. Hence, when we write down the equations describing a theory, each appearance of the covariant derivative $\nabla A$ of a tensor field $A$ is identical to the Christoffel derivative $\widetilde{\nabla} A$ plus interactions between the torsion and $A$. A theory with torsion is thus equivalent to a theory without torsion, plus an extra tensor field with certain couplings. (The fact that the torsion contribution to the connection is a tensor means that it is impossible, when the torsion is nonzero, to choose coordinates in which the coefficients $\Gamma_{\mu \nu}^{\alpha}$ vanish.) In other words, since the torsion transforms as an ordinary tensor field, its status as a "gravitational" field characterizing the geometry of spacetime has the nature of a semantic distinction which we may or may not choose to make. Nevertheless, we will suppose here that a theory of gravitation in which the connection is an independent variable will predict the existence of such a field (whatever we choose to call it), and that there are certain natural interactions for the field to have, given its origin as part of the covariant derivative on the tangent bundle.

With this philosophy in mind, the interaction between torsion and matter fields is straightforward to derive [5,10]. For scalar fields, the covariant derivative is equal to the partial derivative and hence does not involve the connection; there is therefore no interaction between scalars and torsion. The same result holds for gauge fields, although the reasoning is somewhat more subtle. (We are now speaking classically; quantum effects will change the situation, as we discuss below.) We consider for simplicity an abelian gauge field $A_{\mu}$. The only gauge invariant derivative of $A_{\mu}$ we may take is the field strength tensor $F_{\mu \nu}$, which is defined as an exterior derivative: $F \equiv(\mathrm{d} A)$. In components this is the antisymmetric partial derivative,

$$
F_{\mu \nu} \equiv(\mathrm{d} A)_{\mu \nu}=\partial_{\mu} A_{\nu}-\partial_{\nu} A_{\mu} .
$$


While this clearly does not involve the torsion, the mistake is sometimes made of defining the field strength as the antisymmetric covariant derivative. Such a definition suffices when the torsion vanishes, as (2.5) is then recovered; however, with nonzero torsion the antisymmetric covariant derivative induces a gauge non-invariant interaction between the torsion and $A_{\mu}$. There is in fact no reason for such a term to exist, as $(2.5)$ is the correct definition even in curved space (for a full discussion, see [10]).

There is, in contrast, a direct interaction between torsion and fermions. The covariant derivative of a spinor field requires the introduction of the tetrad formalism and a spin connection, which we have been avoiding for simplicity; however, we can transcribe the result, which can be found in the literature [5]. In the presence of torsion the free Dirac Lagrangian for a massive fermion $\psi$ can be decomposed into a torsion-free part $\mathcal{L}_{T F}$ plus an interaction term $\mathcal{L}_{I}$ :

$$
\begin{aligned}
\mathcal{L}_{\text {Dirac }} & =\frac{i}{2}\left(\nabla_{\mu} \bar{\psi} \gamma^{\mu} \psi-\bar{\psi} \gamma^{\mu} \nabla_{\mu} \psi\right)-m \bar{\psi} \psi \\
& =\mathcal{L}_{T F}+\frac{i}{8} T_{\mu \nu \lambda} \bar{\psi} \gamma^{[\mu} \gamma^{\nu} \gamma^{\lambda]} \psi
\end{aligned}
$$

where $\mathcal{L}_{T F}=i \bar{\psi} \gamma^{\mu} \partial_{\mu} \psi-m \bar{\psi} \psi$ and we have set $g_{\mu \nu}=\eta_{\mu \nu}$. Since the gamma matrices are antisymmetrized, only the completely antisymmetric part of the torsion tensor enters the interaction. It is useful to define the vector which is dual to this antisymmetric part:

$$
T^{\sigma} \equiv \frac{1}{3 !} \epsilon^{\mu \nu \lambda \sigma} T_{\mu \nu \lambda}
$$

which can be inverted to yield $T_{[\mu \nu \lambda]}=\epsilon_{\sigma \mu \nu \lambda} T^{\sigma}$. We can then use the identity $\gamma^{\mu} \gamma^{\nu} \gamma^{\lambda} \epsilon_{\mu \nu \lambda \sigma}=$ $(i 3 !) \gamma_{\sigma} \gamma_{5}$ to write the interaction as

$$
\mathcal{L}_{I}=\frac{3}{4} T_{\mu} j_{5}^{\mu}
$$

where we have used the conventional definition of the fermion axial vector current, $j_{5}^{\mu} \equiv$ $\bar{\psi} \gamma^{\mu} \gamma_{5} \psi$.

We see that the entire interaction between torsion and matter reduces to a coupling of the axial vector current to a torsion (pseudo-)vector $T_{\mu}$. In constructing the torsion-only Lagrangian $\mathcal{L}_{T}$, we shall therefore confine our attention to this single vector. While the other components of $T_{\mu \nu}{ }^{\lambda}$ may interact with this vector, they do not couple directly to matter, and are therefore unlikely to yield observable effects. (In what follows we shall treat the vector $T_{\mu}$ as a fundamental field with respect to which we vary the action to obtain equations of motion. In the full theory the fundamental fields would be a vierbein and spin connection.) 
It is straightforward to write down a Lagrangian for the torsion vector which contains all possible terms of no higher than second order in $T_{\mu}$ or derivatives of $T_{\mu}$. We may express it as

$$
\mathcal{L}_{T}=a \partial_{[\mu} T_{\nu]} \partial^{[\mu} T^{\nu]}+b\left(\partial_{\mu} T^{\mu}\right)^{2}+c T_{\mu} T^{\mu} .
$$

A term involving the symmetric part of $\partial_{\mu} T_{\nu}$ can be absorbed, after integration by parts, into the first two terms above. ${ }^{3}$ It is not possible to eliminate $a, b$ or $c$ by a field redefinition, since the interaction (2.8) contains no arbitrary constants. We recall that a vector field describes four degrees of freedom, which can be thought of as a single spin-0 field plus the three polarization modes of a spin-1 field. A simple calculation reveals that it is impossible for both the scalar and the spin-1 components to simultaneously exist as propagating degrees of freedom once we demand that the Hamiltonian of the theory be bounded below. In our notation, this means that the sign of $c$ must be negative for the scalar to be nontachyonic, but positive for the spin-1 field to be non-tachyonic. Hence, in order for our theory to be well-defined, we may choose the parameters $a, b$ and $c$ such that either the scalar or the spin- 1 modes propagate, but not both. ${ }^{4}$ Setting $a=0, b \neq 0$ results in a theory with a propagating scalar, while setting $b=0, a \neq 0$ corresponds to a massive spin1 field. We shall look at the spin-0 theory more closely in the next section. An analysis similar to that given below should be able to provide constraints on the corresponding spin-1 theory.

The theories we consider differ from the conventional Einstein-Cartan theory, defined by

$$
\mathcal{L}_{E C}=-M_{P}^{2} R,
$$

where $R$ is the Ricci scalar $R=g^{\mu \nu} R_{\mu \sigma \nu}^{\sigma}$ and $M_{P}$ is the Planck mass. Upon decomposing $\mathcal{L}_{E C}$ in terms of the metric and torsion, we find that $T_{\mu}$ enters only algebraically:

$$
\mathcal{L}_{E C}=-M_{P}^{2} T_{\mu} T^{\mu}+\text { non }-T_{\mu} \text { terms. }
$$

Einstein-Cartan theory, therefore, corresponds to the choices $a=0, b=0$, and $c=-M_{P}^{2}$ in our equation (2.9). Since the interaction term (2.8) is also free of derivatives of $T_{\mu}$, varying the Einstein-Cartan action with respect to $T_{\mu}$ yields the constraint $T^{\mu}=\left(3 / 8 M_{P}^{2}\right) j_{5}^{\mu}$.

3 We have written (2.9) in terms of $\partial_{\mu}$ rather than $\nabla_{\mu}$, which are not equal when the torsion is nonvanishing. This is justified since the covariant derivative $\nabla_{\mu} T_{\nu}$ is simply the partial derivative $\partial_{\mu} T_{\nu}$ plus interactions between $T_{\mu}$ and other components of the torsion tensor, which we are neglecting by hypothesis.

4 We are, of course, using classical language; in a quantum theory we would say that it is impossible to have four propagating degrees of freedom without involving unphysical ghosts. The problem is present, however, even at the classical level. 
This constraint can then be substituted back into the Lagrangian, resulting in a new fourfermion interaction (suppressed by two powers of the Planck mass). It should be clear that this choice of action leads to no interesting long-range forces; however, we believe that there is no good reason to limit our attention to the Einstein-Cartan action. If the connection is our fundamental variable, it is unnatural to restrict the torsion degrees of freedom such that they do not propagate, unless it is found that theories with torsion are internally inconsistent or in conflict with experiment. We therefore turn to exploration of such a theory.

\section{Consequences}

The spin-0 Lagrangian $\mathcal{L}_{0}$ results from setting $a=0$ in (2.9), and adding the interaction given by (2.8):

$$
\mathcal{L}_{0}=b\left(\partial_{\mu} T^{\mu}\right)^{2}+c T_{\mu} T^{\mu}+\frac{3}{4} T_{\mu} j_{5}^{\mu}
$$

To make the scalar nature of this theory more explicit, we can consider the equivalent expression $\mathcal{L}^{\prime}=\mathcal{L}_{0}+\mathcal{L}_{\lambda}$, where

$$
\mathcal{L}_{\lambda}=-b\left(\lambda-\partial_{\mu} T^{\mu}\right)^{2}
$$

Here, $\lambda$ is a field which functions as a Lagrange multiplier. Varying with respect to $\lambda$ yields the constraint $\lambda=\partial_{\mu} T^{\mu}$; substituting back into (3.2), we find that $\mathcal{L}_{\lambda}$ vanishes, so that $\mathcal{L}_{0}$ and $\mathcal{L}^{\prime}$ define identical theories. However, we may choose instead to keep $\lambda$ in the Lagrangian, and after an integration by parts we obtain

$$
\mathcal{L}^{\prime}=c T_{\mu} T^{\mu}+\frac{3}{4} T_{\mu} j_{5}^{\mu}-b \lambda^{2}-2 b T^{\mu} \partial_{\mu} \lambda .
$$

In this version there are no derivatives of the torsion, and it is $T_{\mu}$ which functions as a Lagrange multiplier (although the physics is, of course, still the same as (3.1)). Variation with respect to $T_{\mu}$ yields the constraint

$$
T_{\mu}=\frac{b}{c} \partial_{\mu} \lambda-\frac{3}{8 c} j_{\mu 5}
$$

We can insert this back into (3.3) to obtain an expression solely in terms of $\lambda$ and $j_{5}^{\mu}$. To make things look conventional, we define $m^{2} \equiv-c / b, f \equiv(-8 c / 9)^{1 / 2}$, and $\phi \equiv$ $\left(-2 b^{2} / c\right)^{1 / 2} \lambda$, which gives

$$
\mathcal{L}^{\prime}=\frac{1}{2}\left(\partial_{\mu} \phi\right)^{2}-\frac{m^{2}}{2} \phi^{2}+\frac{1}{2 f} \phi \partial_{\mu} j_{5}^{\mu}+\frac{1}{8 f^{2}} j_{\mu 5} j_{5}^{\mu} .
$$

Thus, this choice of torsion action is equivalent to a conventional pseudoscalar field with mass $m$ coupled to the divergence of the axial vector current, along with an induced fourfermion interaction. Notice that we must require that $b>0$ and $c<0$ to guarantee that the (mass) $)^{2}$ of $\phi$ be positive and that $f$ be real. 
While the Lagrangian (3.5) specifies the entire classical theory, quantum effects (in the matter sector) will lead to additional interactions. Specifically, there will be an interaction with gauge bosons, mediated by triangle diagrams, due to the chiral anomaly in the current $j_{5}^{\mu}$. The torsion scalar couples to the divergence of $j_{5}^{\mu}$, given by

$$
\partial_{\mu} j_{5}^{\mu}=\frac{N_{f} \alpha}{4 \pi} F_{\mu \nu} \widetilde{F}^{\mu \nu}+\sum_{i} m_{\psi_{i}} \bar{\psi}_{i} \gamma_{5} \psi_{i}
$$

where $F_{\mu \nu}$ is the gauge field strength (we limit our attention to electromagnetism), $\widetilde{F}^{\mu \nu} \equiv$ $\frac{1}{2} \epsilon^{\mu \nu \rho \sigma} F_{\rho \sigma}$ is its dual, $N_{f}$ is the sum of the electric charges of the fermions $\psi_{i}, \alpha$ is the fine structure constant, and $m_{\psi_{i}}$ is the mass of $\psi_{i}$. Equation (3.6) induces an effective interaction Lagrangian $\mathcal{L}_{\phi F \widetilde{F}}$ between torsion and gauge fields:

$$
\mathcal{L}_{\phi F \widetilde{F}}=\frac{N_{f} \alpha}{8 \pi f} \phi F_{\mu \nu} \widetilde{F}^{\mu \nu}
$$

Hence, while there is no interaction between torsion and gauge fields at the classical level, quantum effects (in the form of the chiral anomaly) induce a coupling, which may help to place constraints on the theory.

We now discuss potentially observable consequences of the theory defined by (3.5). In doing this, we may treat the two scales $m$ and $f$ as completely free parameters, and ask what values lead to detectable effects. However, there is good reason to expect that both $m$ and $f$ should be of order the Planck mass $M_{P}$. Indeed, if the Lagrangian includes the Ricci scalar constructed from the connection (the usual Einstein-Cartan choice) as well as pure torsion terms, there is automatically a contribution to $m^{2}$ of order $M_{P}^{2}$, as evidenced by (2.11). Thus, it would require a certain degree of fine tuning for $m$ to be much less than $M_{P}$. Similarly, since $f$ can be though of as $m$ times a dimensionless constant which we would expect to be of order unity, $m \sim f \sim M_{P}$ is a reasonable expectation. However, the resulting theory clearly leads to no observable phenomena. A scalar particle with a mass $M_{P}$ would not be produced in any conceivable experiment; furthermore, it would decay into fermions or gauge bosons with a lifetime $\tau \sim f^{2} / m^{3} \sim M_{P}^{-1}$ [11], so any bosons produced in the early universe would quickly decay away. Thought of as a classical field, the effective range of $\phi$ is also given by $l \sim M_{P}^{-1} \sim 10^{-35} \mathrm{~cm}$; no realistic source would give rise to a long-range field which might be observed. Hence, it is no surprise that torsion has not been detected by any experiment, and the lack of such detection should not be taken as strong evidence that torsion plays no role in the fundamental theory.

While it is reasonable to locate the parameters of the torsion theory in the Planck regime, it is nevertheless possible that they lie at much lower energies. Fortunately, the resemblance of the interactions in (3.5) to previously studied theories allows us to readily 
catalogue the limits on $m$ and $f$. These are presented in Fig. 1. It is clear that the constraints, while interesting, do not approach the Planck scale.

The four-fermion interaction $-\left(1 / 8 f^{2}\right) j_{\mu 5} j_{5}^{\mu}$ allows us to place a limit on $f$, independent of $m$. Such terms have been studied in the context of composite models for quarks and leptons, in which the effective theories often include four-fermi interactions induced at the compositeness scale [12]. The interaction between two axial vector currents is parameterized by a mass scale $\Lambda_{A A}$, related to our $f$ by $f^{2}=\Lambda_{A A}^{2} / 32 \pi$. Constraints on $\Lambda_{A A}$ arise from electron-positron annihilation experiments, in which the four-fermion interaction contributes to a charge asymmetry over and above that expected in the standard model. The best current limits on such an interaction come from $e^{+} e^{-} \rightarrow q \bar{q}$ observations at TRISTAN [13]. These experiments yield a limit $\Lambda_{A A} \geq 3 \mathrm{TeV}$, or

$$
f \geq 3 \times 10^{2} \mathrm{GeV} .
$$

This limit is possible to circumvent, however, by adding a fundamental four-fermion interaction to the initial Lagrangian (3.1). The effect of such a term would be to alter the relation between the torsion coupling constant $f$ and the parameter $\Lambda_{A A}$ governing the strength of the four-fermion interaction, without changing any of the dynamics of the torsion scalar $\phi$. With this in mind, we have indicated the limit (3.8) by a dashed line in Fig. 1.

The interaction of $\phi$ with fermions also leads to constraints from laboratory experiments. The most effective limits come from searches for neutral bosons in $\Upsilon \rightarrow \phi+\gamma$ and $J / \psi \rightarrow \phi+\gamma$ events [14]. Current data enable us to place the limit

$$
m \geq 1 \times 10^{-3} \mathrm{GeV},
$$

which however is only valid for $f \leq 10^{3} \mathrm{GeV}$. This enables us to rule out the bottom left corner of Fig. 1.

Astrophysical effects of the $\phi$ bosons lead to constraints in the $m-f$ plane for somewhat higher values of $f$. The interactions of $\phi$, as specified by (3.5) and (3.7), are precisely those of a pseudo-Goldstone boson (PGB) resulting from the spontaneous breakdown of a global symmetry at a scale $f$, followed by the explicit breakdown of the symmetry at a scale $\Lambda=\sqrt{m f}$. We can use this similarity to our advantage, by applying astrophysical constraints on PGB's to the theory at hand; however, we wish to emphasize that the resemblance is (as far as we know) purely coincidental. There is no spontaneously broken symmetry for which $\phi$ is the pseudo-Goldstone boson; indeed, some of the essential physics is different.

Frieman and Jaffe [11] have presented a comprehensive list of astrophysical constraints on PGB's; we summarize the processes most relevant to our purposes. In the regime 
left unconstrained by ground-based experiments, the most effective bounds come from processes in which PGB's lead to energy loss in stars. Briefly, there may be a range of parameter space in which the mass of $\phi$ is low enough that it can be produced in a stellar interior and the coupling to ordinary matter is sufficiently strong that the rate of production is significant, while at the same time sufficiently weak that the PGB will often escape without further interaction, providing an additional channel for energy loss from the stellar core. Three distinct manifestations of this effect lead to interesting bounds: shortening the lifetime of helium-burning (horizontal branch) stars, preventing helium ignition in lowmass red giants, and shortening the duration of the neutrino pulse from supernova 1987A. The most effective bounds come from SN 1987A; in this case, the coupling of $\phi$ to nucleons can cool the supernova core and noticeably decrease the duration of the neutrino burst. This leads to the constraint

$$
m \geq 6 \times 10^{-2} \mathrm{GeV},
$$

valid for $5 \times 10^{6} \mathrm{GeV} \leq f \leq 10^{10} \mathrm{GeV}$. Larger masses would not affect the supernova, since in that case $m$ would be higher than the characteristic energy of the supernova core. (We are being somewhat loose in quoting these bounds; more precise information can be found in [11].) The resulting gap between $f=10^{3} \mathrm{GeV}$ and $f=5 \times 10^{6} \mathrm{GeV}$ can be closed using the effects on horizontal branch stars and red giants; the former arises because energy loss via the Primakoff process ( $\phi \rightarrow \gamma$ by scattering off electrons or nuclei) decreases the time a star will spend in the helium-burning stage, while the latter results when bremsstrahlung ( $\phi$ production in electron-nucleus scattering) allows red giant cores to cool sufficiently to prevent helium ignition. Taken together, these phenomena lead to the bound

$$
m \geq 2 \times 10^{-5} \mathrm{GeV}
$$

applicable for all $f \leq 10^{9} \mathrm{GeV}$. The astrophysical and laboratory constraints are summarized in Fig. 1. We also note that a seperate set of astrophysical effects are expected for very low-mass or long-range fields; details may be found in [15].

It is important to note that some of the most restrictive cosmological limits on PGB's have no analogue in the torsion theory - specifically, those from production of PGB's in the early universe via cosmic string decay and vacuum misalignment $[11,16]$. In each of these cases the constraint arises because, in certain regions of parameter space, PGB's dominate the energy density of the Universe: $\Omega_{\phi} h^{2} \geq 1$, where $\Omega$ is the density parameter of a Robertson-Walker universe and $h$ is the Hubble constant in units of $100 \mathrm{~km} / \mathrm{sec} / \mathrm{Mpc}$. Determinations of the age of the universe imply that $\Omega_{t o t} h^{2} \leq 1$, leading to limits on $m$ and $f$. However, the production of particles by string decay or vacuum misalignment depends intimately on the nature of $\phi$ as a pseudo-Goldstone boson, the angular degree of freedom in a tilted Mexican hat potential resulting from spontanteous symmetry breaking. 
The torsion theory, in contrast, leads to neither strings nor vacuum misalignment; hence, these constraints are inapplicable. (Conventional thermal production of $\phi$ particles can contribute significantly to the density parameter, but only in a small region of parameter space which is already excluded by the argument from SN1987A.)

\section{Discussion}

We have discussed the empirical constraints on a theory of gravity with a propagating scalar torsion degree of freedom. This theory arises naturally out of a simple set of assumptions, and the associated spin-0 particle is likely to appear in a wide variety of Lagrangians with propagating torsion. Whereas a natural expectation would be for the mass scales characterizing the theory to approach the Planck scale, the region of parameter space accessible to experiment is naturally at much lower energies. From this point of view, it is not surprising that torsion-free general relativity is successful at explaining known observational data.

It is interesting to contrast the theory examined here with other proposals in the literature. Sezgin and van Nieuwenhuizen [9] have studied tacyhon-free gravitational theories, and present five Lagrangians involving the metric and torsion. Their theory 1 is EinsteinCartan theory; theories 2, 4 and 5 propagate the massive scalar particle described in this paper (as well as others); and theory 3 propagates the massive vector particle correponding to the choices $b=0, a \neq 0$ in our Eq. (2.9). They did not discuss observational constraints on their theories, or couplings to matter.

Meanwhile, several papers have considered theories involving a scalar torsion field coupled to $\partial_{\mu} j_{5}^{\mu}$ or $F_{\mu \nu} \widetilde{F}^{\mu \nu}$. An early version of such a theory was proposed by Novello [17], who attempted to couple torsion to electromagnetism in a gauge-invariant fashion. He argued that this was possible if the dual torsion vector was restricted to be the gradient of a scalar, $T_{\mu}=\partial_{\mu} \phi$. A similar proposal was examined in greater detail by De Sabbata and Gasperini [18]. They computed the photon propagator in QED with a constant torsion background, and found that the result was equivalent to the introduction of an effective interaction $T_{\mu} A_{\nu} \widetilde{F}^{\mu \nu}$. On the basis of this result and the desire to preserve gauge invariance, they imposed the restriction that the torsion vector be the gradient of a scalar. The component (2.11) of the Einstein-Cartan Lagrangian involving $T_{\mu}$ then becomes a conventional kinetic term for a scalar field, $\partial_{\mu} \phi \partial^{\mu} \phi$. Since the scalar field only appears in the form $\partial_{\mu} \phi$, it is massless, and the torsion can lead to long-range interactions. In a similar vein, Hammond [19] has proposed an antisymmetric two-index torsion potential $\psi_{\mu \nu}$, related to the torsion tensor by $T_{\mu \nu \sigma}=\partial_{[\sigma} \psi_{\mu \nu]}$ and coupled to electromagnetism through an interaction of the form $F^{\mu \nu} \psi_{\mu \nu}$. Finally, Duncan, Kaloper and Olive [20] have examined Einstein-Cartan theory with the addition of a constraining term $\phi \partial_{\mu} T^{\mu}$, where $\phi$ is treated 
as a Lagrange multiplier. This technique, without imposing any external restrictions on the form of $T_{\mu}$ or $\phi$, introduces a propagating massless scalar field which couples to $\partial_{\mu} j_{5}^{\mu}$.

There are therefore two important distinctions between these investigations of the consequences of scalar torsion theories and the approach advocated in this paper. First, in defining the Lagrangian, we have been able to describe a propagating scalar torsion degree of freedom without imposing external restrictions on the form of the torsion tensor, nor changing the degree-of-freedom content by introducing auxilliary fields as Lagrange multipliers. (In this sense our approach is that of [9].) The second distinction, which is a direct consequence of the first, is that we have found that the resulting scalar should be massive, and indeed with a mass of order the Planck scale. This is not to say that the theories described above are necessarily incorrect; we believe that the approach followed in this paper is simple and natural, but at the current level of understanding this is purely a matter of taste.

The picture of torsion as an extremely short-range field runs somewhat counter to the intuitive conception of torsion as a part of spacetime geometry. More concretely, we are used to gauge theories giving rise to massless, long-range fields, and the status of torsion as part of the connection on the tangent bundle might lead us to expect the same in this case. This conflict with intuition may be resolved by noticing that the torsion is a tensor which is linear in the connection. It therefore becomes possible to construct gauge invariant interactions which give a mass to some of the connection degrees of freedom. This is in contrast with the pure metric theory, or with gauge theories on internal vector bundles, where all gauge invariant terms involve the curvature tensor, constructed from derivatives of the fundamental fields. Thus, despite its origin as part of the geometry of spacetime, the physical manifestation of torsion can be significantly different from that of other "geometrical" fields.

The possible existence of torsion is of interest both in the construction of quantum theories of gravity and in the experimental search for deviations from general relativity. The important lesson of this paper is that the absence of effects of torsion in experiments should not lead us to discount the possibility of torsion playing a role in the ultimate theory of gravity.

\section{Acknowledgments}

It is a pleasure to thank Eric Blackman, Sidney Coleman, Edward Farhi, Roman Jackiw, and Ted Pyne for many useful discussions. This work was supported in part by NASA under Grants No. NAGW-931 and NGT-50850, by the National Science Foundation

under grant PHY/92-06867, and by the U.S. Department of Energy under contract No. DE-FC02-94ER40818. 


\section{References}

1. R. M. Wald, General Relativity (University of Chicago Press, Chicago, 1984).

2. A. Ashtekar, Lectures on Nonperturbative Quantum Gravity (World Scientific, Singapore, 1991).

3. A. Achucarro and P.K. Townsend, Phys. Lett. B229, 383 (1989); E. Witten, Nucl. Phys. B311, 80 (1990).

4. G. Grignani and G. Nardelli, Phys. Rev. D 45, 2719 (1992); D. Cangemi and R. Jackiw, Ann. Phys. 225, 229 (1993); J. D. Romano, Geometrodynamics Vs. Connection Dynamics, preprint UMDGR-93-129, gr-qc/9303032 (1993); P. Peldán, Actions for Gravity, with Generalizations: A Review, Göteborg preprint ITP 93-13, gr-qc/9305011 (1993).

5. F. W. Hehl, P. von der Heyde, G. D. Kerlick, and J. M. Nester, Rev. Mod. Phys. 48, 393 (1976).

6. N. Straumann, General Relativity and Relativistic Astrophysics (Springer-Verlag, Berlin, 1984).

7. C. M. Will, Theory and Experiment in Gravitational Physics (Cambridge Univ. Press, Cambridge, UK, 1993).

8. D. E. Neville, Phys. Rev. D 18, 3535 (1978).

9. E. Sezgin and P. van Nieuwenhuizen, Phys. Rev. D 21, 3269 (1980).

10. I.M. Benn, T. Dereli, and R. W. Tucker, Phys. Lett. 96B, 100 (1980).

11. J. A. Frieman and A. H. Jaffe, Phys. Rev. D 45, 2674 (1992).

12. E. J. Eichten, K. D. Lane, and M. E. Peskin, Phys. Rev. Lett. 50, 811 (1983).

13. K. Abe et al., Phys. Lett. 232B, 425 (1989); I. Adachi et al., Phys. Lett. 255B, 613 (1991).

14. J. E. Kim, Phys. Rep. 150, 2 (1987).

15. S.M. Carroll and G.B. Field, Phys. Rev. D 43, 3789 (1991); D. Harari and P. Sikivie, Phys. Lett. 289B, 67 (1992); S. Mohanty and P.K. Panda, preprint hep-ph/9403205 (1994); W.D. Garretson and E.D. Carlson, preprint (1994).

16. D. Harari and P. Sikivie, Phys. Lett. 195B, 361 (1987); R. Davis and P. Shellard, Nucl. Phys. B324, 167 (1989); A. Dabholkar and J. Quashnock, Nucl. Phys. B333, 815 (1990). 
17. M. Novello, Phys. Lett. 59A, 105 (1976).

18. V. De Sabbata and M. Gasperini, Phys. Lett 77A, 300 (1980); V. De Sabbata and M. Gasperini, Phys. Lett. 83A, 115 (1981).

19. R. T. Hammond, General Relativity and Gravitation 23, 1195 (1991).

20. M. J. Duncan, N. Kaloper and K. A. Olive, Nucl. Phys. B387, 215 (1992). 


\section{Figure Caption}

Figure One. Limits on parameters characterizing the pseudoscalar torsion theory. This theory is specified by two constants $m$ and $f$, each with dimensions of mass. We have plotted the regions excluded by astrophysical and terrestrial data, as explained in the text. The solid lines represent inescapable limits, while the dashed line may be avoided by modification of the induced four-fermion interaction. The star in the upper right represents $m=f=M_{P}=10^{19} \mathrm{GeV}$, which we argued was a reasonable expectation. Clearly, the constraints are far removed from this point. 
This figure "fig1-1.png" is available in "png" format from: http://arXiv.org/ps/gr-qc/9403058v2 